Published in final edited form as:

Transpl Infect Dis. 2018 August ; 20(4): e12902. doi:10.1111/tid.12902.

\title{
Nocardia infections in the transplanted host
}

\author{
M Hemmersbach-Miller, MD, PhD'1 , JE Stout, MD, MHS ${ }^{1}$, MH Woodworth, MD², GM Cox, \\ MD1, and JL Saullo, MD, PharmD ${ }^{1}$ \\ ${ }^{1}$ Division of Infectious Diseases and International Health, Department of Medicine, Duke \\ University Medical Center, Durham, NC, USA \\ 2Department of Medicine, Duke University Medical Center, Durham, NC, USA
}

\begin{abstract}
Background-Nocardia are uncommon pathogens that disproportionately afflict the immunocompromised host. Epidemiology and outcome data of Nocardia infections in transplant recipients are limited.

Methods-We performed a retrospective chart review of all patients at Duke University Hospital with a history of solid organ transplant (SOT) or hematopoietic cell transplant (HCT) and at least one positive culture for Nocardia between 1996-2013. Our aim was to describe the epidemiology and outcomes of Nocardia infections in the transplanted host.
\end{abstract}

Results-During the 18-year study period, 51 patients (14 HCT and 37 SOT recipients) had Nocardia infection. Nocardia incidence was stable during the study period in all populations except heart transplants, whose incidence declined. Infection occurred earlier in the HCT group than the SOT group (median time to diagnosis of 153 and 370 days, respectively). In both groups, the most common site involved was the lung. Outcomes were overall poor, especially in the HCT group with a cure rate of $29 \%$. Heart transplant recipients had significantly better overall survival $(\mathrm{p}<0.05)$ than other patients. Trimethoprim-sulfamethoxazole (TMP-SMX) prophylaxis did not provide complete protection from Nocardia infections, nor did it appear to select for resistant Nocardia isolates.

Conclusions-Infections with Nocardia are typically a late post-transplant complication. The use of TMP-SMX prophylaxis was not associated with TMP-SMX resistant Nocardia. Overall outcomes remain poor.

\section{Keywords}

Nocardia; Transplant Recipients; Hematopoietic Cell Transplantation; Solid Organ Transplantation; Immunocompromised host; Epidemiology

Corresponding author: Marion Hemmersbach-Miller, MD PhD, Duke University Medical Center, Box 102359, Durham, NC, 27710. marion.hemmersbach@dm.duke.edu. Phone: +1-919-668-0789. Fax +1 919 684-8902.

DR MARION HEMMERSBACH-MILLER (Orcid ID : 0000-0001-6041-6815)

DR MICHAEL H. WOODWORTH (Orcid ID : 0000-0002-6181-4599)

\section{Author contributions}

MHM - Drafting article, data collection, data analysis/interpretation, funding. JES - Concept/design, data analysis/interpretation, statistics, critical revision of the article. GMC: Data collection, revision of article. MHW: Data collection, critical revision of article, funding. JLS: Concept/design, data collection, data interpretation, critical revision of article. All authors have approved the final version of the manuscript. 


\section{Introduction}

Nocardia are ubiquitous aerobic gram-positive, partially acid-fast bacilli that can cause localized or disseminated disease, most commonly affecting the lungs, skin and brain (1). They can be found worldwide but some species are more prevalent in specific geographic areas. Transmission most commonly occurs via inhalation or direct inoculation through the skin (2).

Nocardia are uncommon pathogens that disproportionately afflict immunocompromised hosts. Nocardiosis has been described by some as an emerging infectious disease possibly driven by increases in the number of immunocompromised individuals (3). However, it is unclear whether Nocardia incidence is increasing in the transplant population, as some centers report an overall decline in the incidence of Nocardia in comparison to historical data (4). Previously published data from our center demonstrated an increase in Nocardia infections in immunocompetent patients with non-cystic fibrosis-related bronchiectasis and stable to declining Nocardia infections in immunocompromised transplant patients (5).

Epidemiologic and outcome data describing Nocardia infections in the solid organ (SOT) and hematopoietic cell transplant (HCT) populations remain limited (4, 6-12) and many questions exist regarding optimal management. We performed a retrospective review of all SOT and HCT recipients with nocardiosis over an 18-year period at a large academic transplant center to further characterize the presentation, treatment and outcome in this population.

\section{Patients and Methods}

\section{Study Design}

This was a single center, retrospective cohort study of patients seen at Duke University Medical Center, a tertiary care academic center. Duke is an active transplant center with 1660, 1264, 736, 827, 21 and 4584 kidney, lung, liver, heart, heart-lung and HCT transplanted patients, respectively, during the study period. The study was approved by the Duke University Health System Institutional Review Board for Clinical Investigation (Pro00049544).

\section{Data Extraction}

As described in a previously published retrospective cohort study (5), an institutional tool called DEDUCE was used to identify all patients with at least one positive culture for Nocardia over an 18-year period from January $1^{\text {st }}, 1996$ through December $31^{\text {st }}, 2013$ (13). The present study includes a subset of that cohort of patients with a history of either SOT or HCT prior to the positive Nocardia culture. Microbiology and survival data were obtained directly from the DEDUCE system. Patient survival for patients not known to have died during the study period was censored at the date of the last clinic visit within the health care system or at December 31, 2013, whichever came first. All patients who underwent transplantation during the study period were utilized for the incidence calculations. The "atrisk" population was determined utilizing DEDUCE to ascertain the latest encounter in the 
medical system. Clinical data including baseline demographics, underlying disease, transplant status, immunosuppression [including that applied in the presence of rejection or graft-versus host disease (GVHD)], antimicrobial prophylaxis, cytomegalovirus (CMV) coinfection, diagnostic methods, infection characteristics and Nocardia treatment were extracted by manual chart review and entered into a secure Microsoft Access (Microsoft, Redmond, WA) database.

\section{Microbiology}

Nocardia were isolated from specimens sent for bacterial, fungal, and/or mycobacterial culture. Prior to 2006, isolates were typically identified locally using biochemical techniques and antimicrobial susceptibility testing was not routinely done. Since 2006, isolates have been sent to the Mycobacteria/Nocardia Laboratory at the University of Texas, Tyler for species identification which was performed by molecular techniques across the time period, initially with PCR(14) and more recently utilizing partial 16S RNA sequencing. Antimicrobial susceptibility testing was performed utilizing the Clinical and Laboratory Standards Institute (CLSI) recommended broth microdilution minimal inhibitory concentration (MIC) method for Mycobacteria, Nocardiae and other aerobic Actinomycetes.

\section{Definitions}

Nocardia infection was defined as at least one positive culture from a patient specimen. Maintenance immunosuppressive therapies in SOT- and GVHD-directed regimens for HCT recorded at the time of diagnosis of Nocardia infection included prednisone, tacrolimus, cyclosporine, azathioprine, sirolimus or mycophenolate. Corticosteroid dose was calculated as the mean daily dose of prednisone equivalent in the 7 days prior to Nocardia isolation. Antilymphocyte and/or other immunomodulatory use was defined as receipt of one of the following agents within 6 months prior to Nocardia diagnosis: basiliximab or bortezomib; or within 12 months prior to diagnosis: alemtuzumab, daclizumab, muronomab-CD3, thymoglobulin or rituximab.

Sites of disease were identified by a positive culture obtained from that site or positive culture from another site with radiographic evidence of involvement of a secondary site. Disease sites were categorized as lung, skin, brain or other. Disseminated disease was involvement of at least two non-contiguous sites and/or bacteremia. The procedure to diagnose Nocardia was the test by which the first positive Nocardia culture was obtained.

CMV infection and disease were defined as per previously published definitions (15) and within 6 months of the date of Nocardia diagnosis.

Rejection was defined as biopsy-proven rejection of the transplanted solid organ within six months prior to Nocardia diagnosis. GVHD diagnosis in the HCT patients was based on the primary clinician's designation.

Cure was resolution of symptoms with no additional positive cultures. Relapse was recurrence at the site of prior culture-proven or suspected disease. Outcome was classified as cure, death, or unknown. Final outcome data were censored at death, most recent clinic appointment at Duke or December 31, 2013, whichever was soonest. 


\section{Statistical analysis}

Descriptive statistics were used to summarize the basic findings. The chi-square or Fisher's exact test were used to assess associations between categorical variables, as appropriate. Time-to-event analysis was done using the Kaplan Meier method. To compare survival among the groups the log-rank test was used. Fisher's exact test with 2-sided p-values was used to evaluate incidence trends over time. A $p$ value of $<0.05$ was considered statistically significant. Statistical analysis was performed with R (version 3.2.3) using the RStudio interface (version 0.98.1062).

\section{Results}

In total, 51 transplant recipients with Nocardia infection were identified during the 18-year study period, including $14 \mathrm{HCT}$ and 37 SOT recipients. One patient with a remote history of HCT followed by primary lung transplant and ultimate redo lung transplant was included as a SOT recipient for the purpose of this analysis. The lung was the most common site of disease, but many patients with lung involvement also had extrapulmonary disease (Figure 1). Brain imaging (via $\mathrm{CT}$ and/or magnetic resonance imaging) to evaluate for central nervous system (CNS) infection occurred in 41 of the 51 patients (80\%).

Dividing the study into three equal time periods (1996-2001, 2002-2007 and 2008-2013), the incidence of Nocardia infection per 1,000 patients was 28.3, 9 and 7.5 in heart transplants, 3.6, 11.5 and 8.4 in lung transplant recipients and 2.6, 2.0 and 2.4 in HCT patients in the respective time periods. Only the heart transplant patients demonstrated a trend to a significant change with a notable decrease in the incidence of infection across time $(\mathrm{p}=0.069)$.

The primary species of Nocardia isolated was $N$. nova complex, which was found in $39.2 \%$ $(\mathrm{n}=20)$ of the patients, followed by $N$. asteroides complex in $35.3 \%(\mathrm{n}=18)$ and $N$. farcinica in $5.9 \%(\mathrm{n}=3)$. Available antibiotic susceptibilities for Nocardia isolates at our center during this study period have been recently published (5). In this cohort, Nocardia antimicrobial susceptibility testing was not performed on all isolates; however, this testing was available in $86 \%(\mathrm{n}=12)$ and $78 \%(\mathrm{n}=29)$ of HCT and SOT cases respectively. Overall, Nocardia spp isolated from transplant patients were highly susceptible to trimethoprim-sulfamethoxazole (TMP-SMX) (98\%) as well as other agents including linezolid (100\%), amikacin (99\%), imipenem (78\%) and clarithromycin (71\%).

Changes in treatment regimens were common. Twenty-five percent $(n=13)$ received 2 regimens, $14 \%(n=7)$ received 3 regimens and $16 \%(n=8) 4$ or 5 different regimens.

Median length of follow-up after Nocardia diagnosis was 405 days (IQR 64-2350 days) in the SOT group with a total of 50,523 person-days of follow-up and 89 days (IQR 24.3-727 days) in the HCT group with a total of 11,523 patient-days of follow up.

\section{HCT population}

Nocardia infection was identified in 14 patients among 4,584 HCT recipients during the study period. Baseline characteristics of the HCT recipients are shown in Table 1. In general, 
HCT patients were younger than their SOT counterpart (Table 2) with a median age of 38 years (range 1-68 years) and were predominantly male. The majority $(93 \%, \mathrm{n}=13)$ had undergone allogeneic HCT and received a non-myeloablative regimen $(71 \%, \mathrm{n}=10)$, most commonly consisting of alemtuzumab, melphalan and fludarabine. Four patients $(29 \%)$ were receiving TMP-SMX prophylaxis at the time of Nocardia diagnosis. This was a highly immunosuppressed HCT population with 36\% ( $\mathrm{n}=5)$ having undergone at least one prior HCT before the current HCT (either autologous or allogeneic) and 93\% ( $n=13)$ with GVHD at the time of Nocardia diagnosis. Skin GVHD was most common $(85 \%, \mathrm{n}=11)$ followed by GI tract $(69 \%, n=9)$ and liver $(38 \%, n=5)$ involvement with $69 \%(n=9)$ having more than one site involved. Data on management of GVHD was available for $12 / 13$ patients and consisted of at least one agent (range 1-4 agents) including corticosteroids $(100 \%, \mathrm{n}=12$, median daily prednisone dose equivalent of 35mg (IQR 22.5-48.8mg)), calcineurin inhibitors $86 \%$ ( $\mathrm{n}=10$ tacrolimus or cyclosporine), and mycophenolate $(75 \% ; n=9)$. One patient $(7.1 \%)$ had received rituximab and $50 \%(\mathrm{n}=7)$ had received alemtuzumab in the year prior to Nocardia diagnosis. One patient (7.1\%) had received basiliximab in the preceding 6 months.

Characteristics of Nocardia infection in the HCT population are shown in Table 3. Infection occurred a median of 153 days (IQR 134-359 days) following transplantation with the diagnosis made most often within a month of symptom onset. Invasive tests were often utilized to make the diagnosis of Nocardia infection, most commonly bronchoscopy, which was performed in $50 \%(\mathrm{n}=7)$ of patients. Pulmonary infection predominated $(71 \%, \mathrm{n}=10)$ though disseminated infection was common $(50 \%, \mathrm{n}=7)$, including 6 cases $(42.8 \%)$ with bacteremia. $N$. nova complex was the most common species. Of the 12 isolates tested in this group, only one isolate was resistant to TMP-SMX. CMV infection was present in 50\% $(\mathrm{n}=7)$ with only one patient having CMV disease.

The most common initial antibiotic applied was TMP-SMX (93\%, $\mathrm{n}=13)$, followed by carbapenems $(21 \%, \mathrm{n}=3)$ and amikacin $(14 \%, \mathrm{n}=2)$. Fifty-seven percent $(\mathrm{n}=8)$ of treated HCT patients received monotherapy. Treatment was modified due to either intolerance or disease progression in $21 \%(\mathrm{n}=3)$ and $14 \%(\mathrm{n}=2)$ of patients, respectively. In patients who were cured $(n=4,29 \%)$, median duration of treatment was 289 days (IQR 240-339 days). Only one patient (25\%) was placed on secondary prophylaxis following completion of therapy. Treatment outcomes were overall poor (Figure 2). Seventy-one percent of patients $(\mathrm{n}=10)$ died prior to completion of Nocardia-directed therapy, with $50 \%(\mathrm{n}=7)$ of the patients dying within 3 months of Nocardia infection. Of those cured of infection no relapse was identified.

\section{SOT population}

Nocardia infection was identified in 37 SOT patients during the study period. Baseline demographics are shown in Table 2. Immunosuppression regimens in SOT recipients consisted of prednisone $(97 \%, \mathrm{n}=36)$, tacrolimus $(78 \%, \mathrm{n}=29)$, cyclosporine $(19 \%, \mathrm{n}=7)$ and mycophenolate mofetil or azathioprine $(68 \%, \mathrm{n}=25)$. The median daily prednisone dose at time of diagnosis of the Nocardia infection was $10 \mathrm{mg}$ (IQR 7.5-20mg).

Eleven percent of patients $(n=4)$ had received basiliximab in the preceding 6 months. Five percent of patients $(n=2)$ had received alemtuzumab and one patient $(3 \%)$ had received 
muromonab-CD3 in the preceding 12 months. Rejection was seen in $24 \%(\mathrm{n}=9)$ of the patients in the preceding 6-months. CMV infection was present in $24 \%(\mathrm{n}=9)$ of the SOT patients, however only one patient had CMV disease.

Characteristics of Nocardia infection in the SOT population are shown in Table 3. Infection occurred a median of 370 days (IQR 141-885 days) following transplantation with the diagnosis made after a longer symptomatic period when compared to the HCT group. Similar to HCT, bronchoscopic evaluation was the primary diagnostic procedure; lung involvement predominated in the SOT group but disseminated disease was common (27\%, $\mathrm{n}=10)$ including $6(16.2 \%)$ cases with bacteremia, albeit less common than in the HCT group. $N$. nova complex also predominated in SOT recipients.

The most common initial antibiotic applied was TMP-SMX $(81 \%, \mathrm{n}=30)$ followed in frequency by a carbapenem $(38 \%, n=14)$. Monotherapy was used in $43 \%(n=16)$. Treatment was modified due to either intolerance or disease progression in $16 \%(\mathrm{n}=6)$ and $3 \%(\mathrm{n}=1)$ of patients, respectively. Of the 29 Nocardia isolates tested, none were resistant to TMP-SMX. Nocardia infections were treated a median duration of 348 days (IQR 234-391 days) and $60 \%(\mathrm{n}=15)$ of patients were placed on secondary prophylaxis following completion of therapy. TMP-SMX was the most commonly employed agent for prophylaxis although 2 patients $(5 \%)$ received azithromycin due to allergy or intolerance of TMP-SMX. Treatment outcomes were better in the SOT than the HCT group with 52\% $(\mathrm{n}=19)$ of SOT recipients cured, $32 \%(\mathrm{n}=12)$ dying prior to completion of Nocardia-directed therapy and 2 patients (5\%) with relapsed disease. Patients with heart transplants had significantly better survival than all other groups $(\mathrm{p}=0.00064)$ (Figure 2$)$.

\section{Discussion}

We report 51 cases of Nocardia infection occurring in 14 HCT and 37 SOT recipients diagnosed at a single tertiary transplant center over an 18 -year period. The literature to date regarding nocardiosis in transplant consists primarily of retrospective case series or reports $(4,6-10)$ alongside a single center retrospective case control study in SOT patients in the United Sates (11) and a more contemporary multicenter case-control study in SOT patients in Europe $(10,12)$. This study adds to the limited data that exists regarding nocardiosis following transplant.

Despite an overall increase in the immunocompromised population over time and concerns that Nocardia represents an emerging pathogen in transplantation (3), we did not see significant increases in the incidence across time in our SOT or HCT groups. There were no reported Nocardia infections in our pancreas, intestinal or multivisceral transplant populations. Moreover, consistent with other centers (16-18) we found a trend towards a decrease in the incidence among the heart transplant population from 28/1000 patients in the 1996-2001 time period to 7.5/1000 patients in 2008-2013. Though not statistically significant, the incidence of nocardiosis in the lung transplant population increased overall from the earliest time period aligning with more recent data from other centers wherein nocardiosis has been reported most commonly in this SOT group $(11,19)$. Plausible explanations for this include the high level of immunosuppression applied in this population, 
an increase in lung transplantation over time alongside the unique scenario of direct exposure of the allograft to the outside environment with an organism most commonly acquired via the inhalational route.

Multiple questions exist regarding the optimal management of Nocardia infections including the appropriate duration of therapy as well as the utility of primary and secondary prophylaxis. An extended treatment course is typically recommended in the immunocompromised host including 6 to 12-months of therapy for pulmonary infections and 12-months for CNS and/or disseminated infections $(1,8)$ given concerns for relapsed or recurrent infection with shorter treatment courses (20-22). The median duration of therapy applied at our center in patients with an initial outcome of cure was 289 (IQR 240-339) and 348 (IQR 234-391) days in the HCT and SOT group, respectively, and consistent with these recommendations. More recent studies have suggested that shorter courses of therapy (e.g., $<120$ days) may be effective in treating Nocardia $(12,23)$ without relapsed disease. However, this data remains limited and short-duration therapy merits further investigation before acceptance into routine practice. Our study did not include sufficient numbers of patients receiving short-course therapy to compare outcomes to patients receiving longer courses. The role of primary and secondary prophylaxis is perhaps even less straightforward. TMP-SMX is often employed for variable periods in the post-transplant period as primary prophylaxis for Pneumocystis jiroveci and Toxoplasmosis. Historical data regarding the impact of TMP-SMX prophylaxis on Nocardia prevention in SOT suggested a protective effect $(24,25)$ whereas larger contemporary case-control series demonstrated the emergence of Nocardia infection despite receipt of prophylaxis in 18 to $69 \%$ of patients $(10,11)$. Similar to the SOT population, breakthrough Nocardia infection during TMP-SMX prophylaxis has been described in the HCT population $(7,26)$. Some have argued that although Nocardia prophylaxis with TMP-SMX is incompletely protective, it may reduce the severity of infection (e.g., prevent or delay the development of dissemination) (27). In our cohort, TMP-SMX susceptible Nocardia infections occurred despite the prescription of TMP-SMX prophylaxis in 37.3\% $(\mathrm{n}=19)$ of cases overall. Multiple explanations for "breakthrough infection" despite TMP-SMX prophylaxis may exist including suboptimal dosing, impaired absorption and/or non-compliance. Due to the small sample size and lack of a control group we cannot make any conclusions regarding the effectiveness of TMPSMX prophylaxis. The single patient in this series with a TMP-SMX-resistant Nocardia isolate was not taking TMP-SMX prophylaxis. Hence, we did not see an association between TMP-SMX prophylaxis and emergence of resistance.

When considering long-term management of these patients, secondary prophylaxis is often employed in those with ongoing immunosuppression due to the concern for recurrent infection $(8,20,21,25)$. Delineating the most appropriate secondary prophylactic agent and dose may become more important if further data support shorter courses of primary therapy. In our series, secondary prophylaxis was prescribed in $31 \%$ of patients, most commonly with TMP-SMX. No standard approach to prophylactic dosing was observed, with doses ranging from $80 \mathrm{mg}$ of the TMP component three times a week to $240 \mathrm{mg}$ per day in patients with varying weight and renal function. Two patients in our series were prescribed azithromycin for secondary prophylaxis in isolates deemed susceptible to clarithromycin; neither of these patients developed a Nocardia relapse. Data regarding the use of 
azithromycin for the management of Nocardia is overall limited but warrants consideration given poor tolerability of TMP-SMX in this population.

Relapse of Nocardia infection was found in two patients in our cohort, one with prior lung transplant and the second with prior heart transplant. Neither received short-course antibiotic therapy. One relapse occurred off TMP-SMX prophylaxis, as the patient had reportedly been intolerant to sulfa drugs and presented with disseminated Nocardia infection. After treatment of the relapse, secondary prophylaxis was continued with no further recurrence of Nocardia infection. Data on secondary prophylaxis are not available on the second patient that relapsed. This patient developed chronic rejection and died two years after the relapsed Nocardia infection.

Overall, outcomes were poor in this series. Within the SOT group, $57 \%$ were cured compared to $89 \%$ in a comparable U.S-based single center study (11). Among the SOT groups, heart transplant recipients had a significantly better overall survival $(\mathrm{p}<0.05)$ than any of the other subgroups (Figure 2). Consistent with the literature and our series, historically, heart transplants have had a higher incidence of Nocardia infection. This trend has improved over time, in part, due to advances in immunosuppressive regimens applied $(16,18)$. It is unclear why improvements in immunosuppressive regimens for heart transplant recipients would confer differential benefit compared to other SOT groups with presumably equal access to these improvements. A change in prophylactic practices in the heart transplant population across time may have also contributed to this trend; however, this was not directly evaluated in this study. Cure rates were particularly low in the HCT subgroup with only $29 \%$ of HCT patients cured of infection. These rates are worse compared to outcomes reported by Shannon et al. with complete disease resolution in 57\% (7). This could be influenced by multiple factors including underlying disease, comorbidities as well as post-transplant complications such as GVHD and infections with other pathogens. Notably, HCT patients in our series had a high degree of immunosuppression, $93 \%$ of whom had GVHD at the time of Nocardia diagnosis.

\section{Limitations}

Limitations of this study are the retrospective and single center design including the inability to capture all Nocardia infections occurring outside of our institution. Also, patients transplanted prior to the study period were not included in the "at-risk" population when evaluating the incidence of infection across time and may have impacted these results. In addition, molecular species identification was only performed in 29 out of 51 (56.8\%) patients, thus many of the isolates classified as $N$. asteroides complex prior to utilization of molecular techniques would likely be reclassified (2) with newer techniques hence overestimating the true percentage of these infections. Furthermore, no differentiation between colonization and infection of Nocardia was made in this study. While this is an interesting concept mentioned elsewhere (28), it is important to point out that colonization is more frequently found in immunocompetent patients. Taking into account isolation sources, clinical characteristics, radiologic abnormalities and pathology results, Wang et al. (29) tried to categorize Nocardia infections as definite, probable or doubtful infections, with the latter representing colonization or contamination. Although the general approach is a valid

Transpl Infect Dis. Author manuscript; available in PMC 2019 August 01. 
suggestion, definitions such as those proposed by the European Organization for Research and Treatment of Cancer/Invasive Fungal Infections Cooperative Group and the National Institute of Allergy and Infectious Diseases Mycoses Study Group (EORTC/MSG) available for invasive fungal diseases (30) are lacking for Nocardia infection. Additionally, the severe and often disseminated infections associated with Nocardia in transplant recipients support prompt initiation of therapy when isolated.

\section{Conclusion}

In conclusion, we present the epidemiology and outcome of Nocardia infections in HCT and SOT recipients at a single center from 1996-2013. Due to the nature of the disease, invasive diagnostics were often needed. The use of TMP-SMX prophylaxis did not appear to result in TMP-SMX resistant Nocardia spp. Susceptibility testing remains imperative as results can vary by species. Outcomes remain poor, likely driven by the severity of underlying disease in this population.

Clinicians should maintain a high degree of suspicion for this pathogen that usually presents months to years after transplant given the potential for severe infection necessitating specific antimicrobial therapy and management. Further study of risk factors for Nocardia infection in transplant patients and the optimal management strategies, including primary and secondary prophylaxis would be valuable.

\section{Acknowledgments}

Funding

Research reported in this publication was supported by the National Institute of Allergy and Infectious Diseases (NIAID) of the National Institutes of Health under award number 5T32AI100851 (MHM) and by a Duke Internal Medicine Grant (MHW). The content is solely the responsibility of the authors and does not necessarily represent the official views of the National Institutes of Health.

The authors thank Barbara Brown-Elliott, MS, MT (ASCP) SM from the Mycobacteria/Nocardia Laboratory at the University of Texas Health Science Center for her assistance in delineating molecular methods used for Nocardia identification over the study period.

\section{References}

1. Lerner PI. Nocardiosis. Clin Infect Dis. 1996; 22(6):891-903. quiz 4-5. [PubMed: 8783685]

2. Brown-Elliott BA, Brown JM, Conville PS, Wallace RJ Jr. Clinical and laboratory features of the Nocardia spp. based on current molecular taxonomy. Clin Microbiology Rev. 2006; 19(2):259-82.

3. Ambrosioni J, Lew D, Garbino J. Nocardiosis: updated clinical review and experience at a tertiary center. Infection. 2010; 38(2):89-97. [PubMed: 20306281]

4. Lebeaux D, Morelon E, Suarez F, Lanternier F, Scemla A, Frange P, et al. Nocardiosis in transplant recipients. Eur J Clin Microgiol Infect Dis. 2014; 33(5):689-702.

5. Woodworth MH, Saullo JL, Lantos PM, Cox GM, Stout JE. Increasing Nocardia Incidence Associated with Bronchiectasis at a Tertiary Care Center. Ann Am Thorac Soc. 2017; 14(3):347-54. [PubMed: 28231023]

6. Mansi L, Daguindau E, Saas P, Pouthier F, Ferrand C, Dormoy A, et al. Diagnosis and management of nocardiosis after bone marrow stem cell transplantation in adults: lack of lymphocyte recovery as a major contributing factor. Pathol Biol. 2014; 62(3):156-61. [PubMed: 24875455]

7. Shannon K, Pasikhova Y, Ibekweh Q, Ludlow S, Baluch A. Nocardiosis following hematopoietic stem cell transplantation. Transpl Infect Dis. 2016; 18(2):169-75. [PubMed: 26809666] 
8. Clark NM, Reid GE. Nocardia infections in solid organ transplantation. Am J Transplant. 2013; 13(Suppl 4):83-92. [PubMed: 23465002]

9. Wiesmayr S, Stelzmueller I, Tabarelli W, Bargehr D, Graziadei I, Freund M, et al. Nocardiosis following solid organ transplantation: a single-centre experience. Transplant international. 2005; 18(9):1048-53. [PubMed: 16101725]

10. Coussement J, Lebeaux D, van Delden C, Guillot H, Freund R, Marbus S, et al. Nocardia Infection in Solid Organ Transplant Recipients: A Multicenter European Case-control Study. Clin Infect Dis. 2016; 63(3):338-45. [PubMed: 27090987]

11. Peleg AY, Husain S, Qureshi ZA, Silveira FP, Sarumi M, Shutt KA, et al. Risk factors, clinical characteristics, and outcome of Nocardia infection in organ transplant recipients: a matched casecontrol study. Clin Infect Dis. 2007; 44(10):1307-14. [PubMed: 17443467]

12. Lebeaux D, Freund R, van Delden C, Guillot H, Marbus SD, Matignon M, et al. Outcome and Treatment of Nocardiosis After Solid Organ Transplantation: New Insights From a European Study. Clin Infect Dis. 2017; 64(10):1396-405. [PubMed: 28329348]

13. Horvath MM, Rusincovitch SA, Brinson S, Shang HC, Evans S, Ferranti JM. Modular design, application architecture, and usage of a self-service model for enterprise data delivery: the Duke Enterprise Data Unified Content Explorer (DEDUCE). J Biomed Inform. 2014; 52:231-42. [PubMed: 25051403]

14. Steingrube VA, Brown BA, Gibson JL, Wilson RW, Brown J, Blacklock Z, et al. DNA amplification and restriction endonuclease analysis for differentiation of 12 species and taxa of Nocardia, including recognition of four new taxa within the Nocardia asteroides complex. J Clin Microbiol. 1995; 33(12):3096-101. [PubMed: 8586680]

15. Ljungman P, Boeckh M, Hirsch HH, Josephson F, Lundgren J, Nichols G, et al. Definitions of Cytomegalovirus Infection and Disease in Transplant Patients for Use in Clinical Trials. Clin Infect Dis. 2016

16. Haddad F, Deuse T, Pham M, Khazanie P, Rosso F, Luikart H, et al. Changing trends in infectious disease in heart transplantation. J Heart Lung Transplant. 2010; 29(3):306-15. [PubMed: 19853478]

17. Peraira JR, Segovia J, Fuentes R, Jimenez-Mazuecos J, Arroyo R, Fuertes B, et al. Pulmonary nocardiosis in heart transplant recipients: treatment and outcome. Transplant Proc. 2003; 35(5): 2006-8. [PubMed: 12962878]

18. Koerner MM, El-Banayosy A, Schulz U, Zeriouh M, Koerfer R, Tenderich G, et al. Nocardiosis in Heart Transplant Recipients. Heart Surg Forum. 2015; 18(6):E250-2. [PubMed: 26726715]

19. Santos M, Gil-Brusola A, Morales P. Infection by Nocardia in solid organ transplantation: thirty years of experience. Transplant Proc. 2011; 43(6):2141-4. [PubMed: 21839216]

20. King CT, Chapman SW, Butkus DE. Recurrent nocardiosis in a renal transplant recipient. Southern Med J. 1993; 86(2):225-8. [PubMed: 8434299]

21. Palmer DL, Harvey RL, Wheeler JK. Diagnostic and therapeutic considerations in Nocardia asteroides infection. Medicine. 1974; 53(5):391-401. [PubMed: 4604319]

22. Wallace RJ Jr, Septimus EJ, Williams TW Jr, Conklin RH, Satterwhite TK, Bushby MB, et al. Use of trimethoprim-sulfamethoxazole for treatment of infections due to Nocardia. Rev Infect Dis. 1982; 4(2):315-25. [PubMed: 6981158]

23. Tripodi MF, Durante-Mangoni E, Fortunato R, Cuccurullo S, Mikami Y, Farina C, et al. In vitro activity of multiple antibiotic combinations against Nocardia: relationship with a short-term treatment strategy in heart transplant recipients with pulmonary nocardiosis. Transpl Infect Dis. 2011; 13(4):335-43. [PubMed: 21156012]

24. Peterson PK, Ferguson R, Fryd DS, Balfour HH Jr, Rynasiewicz J, Simmons RL. Infectious diseases in hospitalized renal transplant recipients: a prospective study of a complex and evolving problem. Medicine. 1982; 61(6):360-72. [PubMed: 6292652]

25. Wilson JP, Turner HR, Kirchner KA, Chapman SW. Nocardial infections in renal transplant recipients. Medicine. 1989; 68(1):38-57. [PubMed: 2642586]

26. van Burik JA, Hackman RC, Nadeem SQ, Hiemenz JW, White MH, Flowers ME, et al. Nocardiosis after bone marrow transplantation: a retrospective study. Clin Infect Dis. 1997; 24(6): 1154-60. [PubMed: 9195074] 
27. Freites V, Sumoza A, Bisotti R, Mujica M, Cabrera A, Costa M, et al. Subcutaneous Nocardia asteroides abscess in a bone marrow transplant recipient. Bone Marrow Transplant. 1995; 15(1): 135-6. [PubMed: 7742746]

28. Fujita T, Ikari J, Watanabe A, Tatsumi K. Clinical characteristics of pulmonary nocardiosis in immunocompetent patients. J Infect Chemother. 2016; 22(11):738-743. [PubMed: 27615155]

29. Wang HL, Seo YH, LaSala PR, Tarrand JJ, Han XY. Nocardiosis in 132 patients with cancer: microbiological and clinical analyses. Am J Clin Pathol. 2014; 142(4):513-23. [PubMed: 25239419]

30. De Pauw B, Walsh TJ, Donnelly JP, Stevens DA, Edwards JE, Calandra T, et al. Revised Definitions of Invasive Fungal Disease from the European Organization for Research and Treatment of Cancer/Invasive Fungal Infections Cooperative Group and the National Institute of Allergy and Infectious Diseases Mycoses Study Group (EORTC/MSG) Consensus Group. Clin Infect Dis. 2008; 46(12):1813-21. [PubMed: 18462102] 


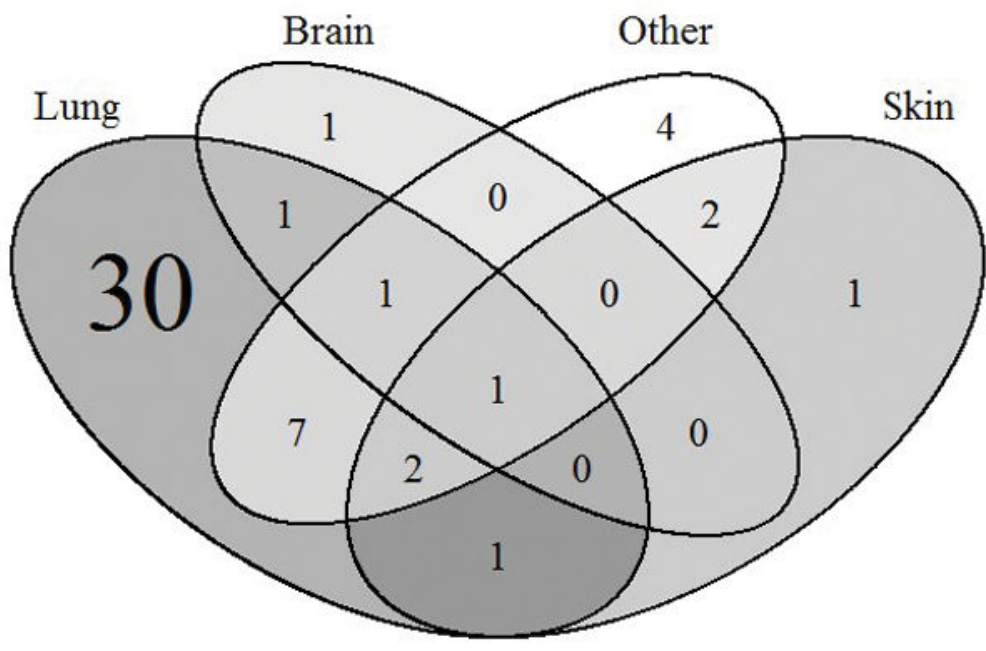

Figure 1.

Sites of Nocardia infection in transplant patients. Venn diagram. 


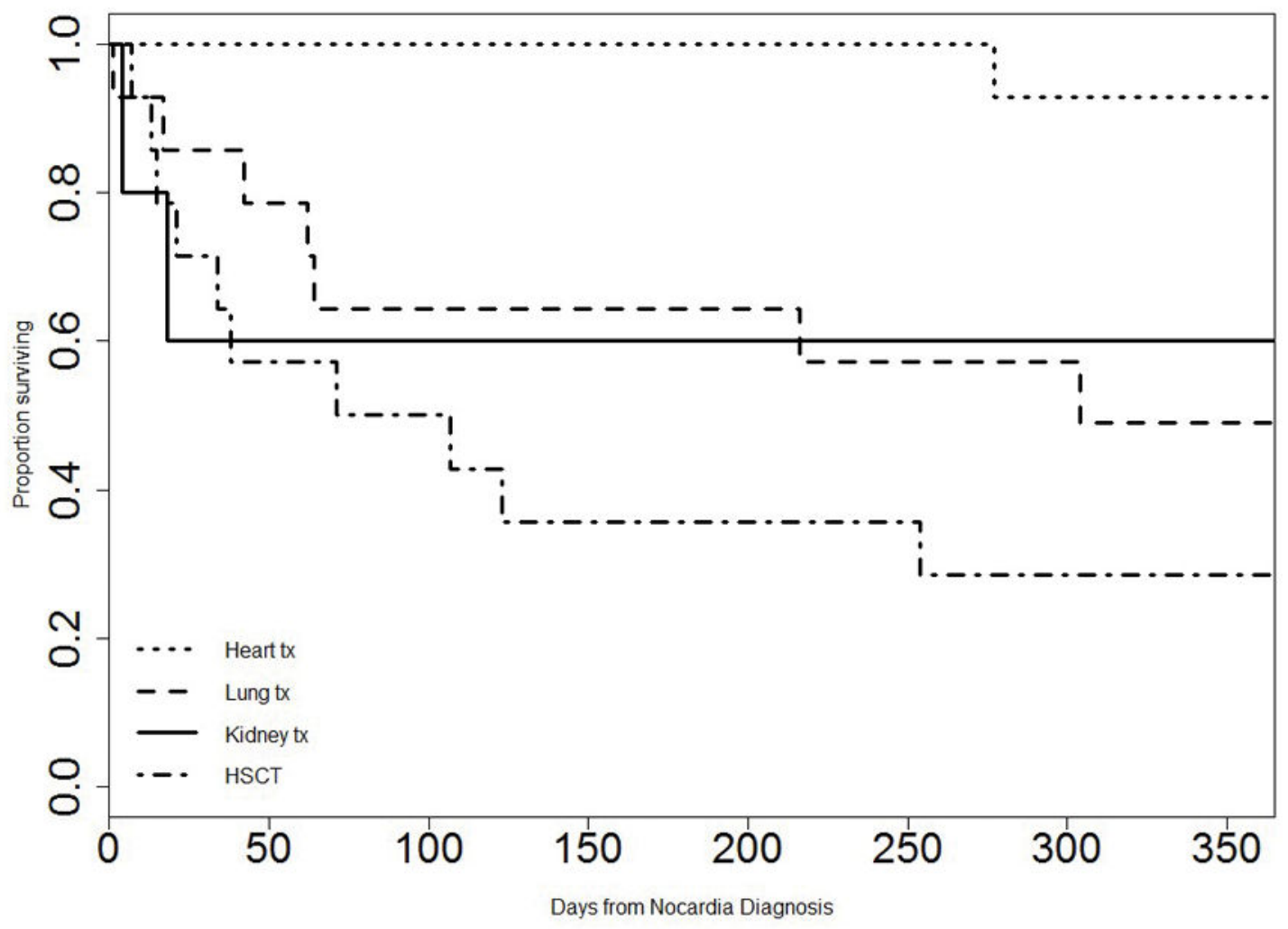

Figure 2.

Kaplan Meier one -year survival curve after Nocardia infection diagnosis among transplant recipients.

Tx: transplant; HSCT: hematopoietic cell transplant. Global differences among represented subgroups $(\mathrm{p}=0.005)$. Survival difference between heart transplant recipients and all other subgroups $(\mathrm{p}=0.00064)$. 


\section{Table 1}

Demographic and transplant characteristics of patients with Nocardia infection and preceding HCT, 19962013. $n=14$.

\begin{tabular}{|c|c|}
\hline Median age in years (range) & $38(1-68)$ \\
\hline Female n $(\%)$ & $3(21)$ \\
\hline \multicolumn{2}{|l|}{ Race/ethnicity n (\%) } \\
\hline Black, non-Hispanic & $2(14)$ \\
\hline White, non-Hispanic & $12(86)$ \\
\hline \multicolumn{2}{|l|}{ Underlying Disease n (\%) } \\
\hline Acute myelogenous leukemia & $1(7)$ \\
\hline Chronic lymphocytic leukemia & $1(7)$ \\
\hline Chronic myelogenous leukemia & $1(7)$ \\
\hline Hodgkin Lymphoma & $4(29)$ \\
\hline Non-Hodgkin lymphoma & $1(7)$ \\
\hline Myelodysplastic syndrome & $1(7)$ \\
\hline Multiple Myeloma & $1(7)$ \\
\hline Other (PNH, WAS) & $2(14)$ \\
\hline \multicolumn{2}{|l|}{ Donor Source n (\%) } \\
\hline Autologous & $1(7)$ \\
\hline Matched related donor & $5(36)$ \\
\hline Matched unrelated donor & $7(50)$ \\
\hline Umbilical cord donor & $1(7)$ \\
\hline \multicolumn{2}{|l|}{ Conditioning Regimen n (\%) } \\
\hline Myeloablative & $4(29)$ \\
\hline Non-myeloablative & $10(71)$ \\
\hline
\end{tabular}

PNH: paroxysmal nocturnal hemoglobinuria; WAS: Wiskott Aldrich Syndrome. 
Table 2

Demographics and transplant characteristics of patients with Nocardia infection and preceding SOT, 19962013. $n=37$.

\begin{tabular}{|l|c|}
\hline Median age in years (range) & $59(19-73)$ \\
\hline Female n (\%) & $10(27)$ \\
\hline Race/ethnicity n (\%) & $11(30)$ \\
Black, non-Hispanic & $25(68)$ \\
White, non-Hispanic & $1(3)$ \\
Other/unknown & \\
\hline Underlying disease n (\%) & $10(27)$ \\
Ischemic heart disease & $5(13.5)$ \\
Non ischemic heart disease & $3(8.1)$ \\
Chronic obstructive pulmonary disease & $6(16)$ \\
Pulmonary fibrosis & $1(3)$ \\
Cystic fibrosis & $1(3)$ \\
Hypertension & $1(3)$ \\
Polycystic kidney disease & $2(5)$ \\
Autoimmune kidney disease & $8(22)$ \\
Other & $15(40.5)$ \\
\hline Transplant type & $1(3)$ \\
Heart & $5(13.5)$ \\
Heart-lung & $1(3)$ \\
Kidney & $15(40.5)$ \\
Liver & \\
Lung & \\
\hline
\end{tabular}


Table 3

Clinical characteristics of Nocardia infection in HCT and SOT recipients.

\begin{tabular}{|c|c|c|}
\hline & $\underset{n=14}{\operatorname{HCT} n(\%)}$ & $\underset{\mathbf{n}=37}{\operatorname{SOT} \mathbf{n}(\%)}$ \\
\hline \multicolumn{3}{|l|}{ Symptom duration prior to diagnosis } \\
\hline Less than one week & $6(43)$ & $4(11)$ \\
\hline 1 week -1 month & $2(14)$ & $15(41)$ \\
\hline $1-3$ months & $3(21)$ & $7(19)$ \\
\hline$>3$ months & $1(7)$ & $3(8)$ \\
\hline Unknown & $2(14)$ & $8(22)$ \\
\hline Time from most recent transplant to diagnosis (median days, range) & $153(33-1054)$ & $370(0-2920)$ \\
\hline \multicolumn{3}{|l|}{ Sites of infection } \\
\hline Lung & $10(71)$ & $33(89)$ \\
\hline Brain & $1(8)$ & $3(8)$ \\
\hline Skin & $1(8)$ & $6(16)$ \\
\hline Disseminated (includes bacteremia) & $7(50)$ & $10(27)$ \\
\hline With bacteremia & $6(43)$ & $6(16)$ \\
\hline \multicolumn{3}{|l|}{ Procedure used to diagnose } \\
\hline \multicolumn{3}{|l|}{ Nocardia infection } \\
\hline Bronchoscopy & $7(50)$ & $15(41)$ \\
\hline Blood culture & 0 & $1(3)$ \\
\hline Craniotomy & $1(7)$ & 0 \\
\hline Open lung biopsy & $2(14)$ & $4(11)$ \\
\hline Other open biopsy & $2(14)$ & $4(11)$ \\
\hline Radiographically-guided needle biopsy & $1(7)$ & $5(14)$ \\
\hline Skin biopsy & $1(7)$ & $2(5)$ \\
\hline Sputum culture & 0 & $6(16)$ \\
\hline \multicolumn{3}{|l|}{ Cytomegalovirus (CMV) } \\
\hline CMV Infection & $7(50)$ & $9(24)$ \\
\hline CMV disease & $1(7)$ & $1(3)$ \\
\hline
\end{tabular}

